\title{
Article
}

\section{Three-dimensional kinematic differences between accurate and high velocity kicks in rugby union place kicking}

Sinclair, Jonathan Kenneth, Smith, Adam, Bullen, Joe, Taylor, Paul John and Hobbs, Sarah Jane

Available at http://clok.uclan.ac.uk/15338/

Sinclair, Jonathan Kenneth ORCID: 0000-0002-2231-3732, Smith, Adam, Bullen, Joe, Taylor, Paul John ORCID: 0000-0002-9999-8397 and Hobbs, Sarah Jane ORCID: 0000-0002-1552-8647 (2017) Three-dimensional kinematic differences between accurate and high velocity kicks in rugby union place kicking. International Journal of Sports Science and Coaching, 12 (3). pp. 371380. ISSN 1747-9541

It is advisable to refer to the publisher's version if you intend to cite from the work. http://dx.doi.org/10.1177/1747954117710515

For more information about UCLan's research in this area go to

http://www.uclan.ac.uk/researchgroups/ and search for <name of research Group>.

For information about Research generally at UCLan please go to http://www.uclan.ac.uk/research/

All outputs in CLoK are protected by Intellectual Property Rights law, including Copyright law. Copyright, IPR and Moral Rights for the works on this site are retained by the individual authors and/or other copyright owners. Terms and conditions for use of this material are defined in the policies page. 


\title{
Three-dimensional kinematic differences between accurate and high
} velocity kicks in rugby union place kicking.

\author{
${ }^{1}$ Sinclair J, ${ }^{2}$ Taylor P.J, ${ }^{1}$ Smith A, ${ }^{1}$ Bullen J, ${ }^{1}$ Bentley I, ${ }^{1}$ Hobbs S.J
}

1. Centre for Applied Sport and Exercise Sciences, College of Health \& Wellbeing, University of Central Lancashire

2. School of Psychology, College of Science \& Technology, University of Central Lancashire

\section{Contact Details:}

Jonathan Sinclair,

University of Central Lancashire,

Preston,

PR1 2HE.

e-mail: JKSinclair@uclan.ac.uk

Keywords: rugby, kicking, kinematics, ball velocity.

Word count: 3150

\section{Abstract}

Place kicking occurs many times during a rugby union game with more than half of all points scored coming from place kicking. Ball velocity is an important biomechanical indicator of kicking success but it also evident that the ball must be kicked accurately to pass between the posts. This study aimed to identify biomechanical differences in rugby place kicking kinematics when kicking towards a specific target and for maximum velocity. Ten male 
rugby union kickers performed place kicks in two conditions 1 . for maximum velocity and 2 . towards a pre-defined target. Lower extremity kinematics were obtained using an optoelectric motion capture system operating at $500 \mathrm{~Hz}$. Differences in lower extremity kinematics between the two kicking conditions were examined using paired t-tests. Higher ball velocities were obtained when kicking for maximum velocity. Foot linear velocity, knee extension velocity and hip extension velocity were also found to be greater when kicking for maximum velocity. Ankle dorsiflexion and peak external rotation were found to be greater in the accuracy condition. The findings suggest that rugby kickers may have selected distinct kicking mechanics characterised by reduced joint angular velocities and a more externally rotated foot position in a deliberate attempt to improve precision, sacrificing ball velocity and thus the distance that the ball can be kicked. The specific findings from the current work have implications for coaches and applied practitioners which may facilitate improvements in kicking performance.

\section{Introduction}

Place kicking is used frequently during rugby union games (Baktash et al., 2009), and is now a key determinant of success in the modern game. Of the total points scored in 2012 by the highest seeded international sides, more than half of all points scored come from place kicking either in the form of a conversion or a penalty (Sinclair et al., 2014).

Like soccer instep kicks, place kicks involve a series of motions that include an initial address to the ball, planting of the support leg beside the ball, and striking of the ball with the instep of the kicking foot (Barfield 1995; Lees \& Nolan 1998). Whilst the basic mechanical actions of the place kick are similar to the instep kick in soccer players, differences in ball shape, tee support, and release angles make the place kicking technique unique (Baktash et al., 2009; Bezodis et al., 2009; Zhang et al., 2011).

\section{The release velocity of the ball is considered the main biomechanical indicator of kicking} success in most sports that involve stationary kicking and it is the result of various factors, including technique (Lees \& Nolan, 1998). As such place kicking for maximal resultant ball 
velocity is desirable, particularly in modern rugby union where kicks in excess of $50 \mathrm{~m}$ are not uncommon (Zhang et al., 2011). Nonetheless, whilst it is clear that the ball must be struck with sufficient velocity to reach the posts it is also evident that the ball must be kicked accurately in order for it to pass between the posts to allow the points to register. The analysis of accurate kicks has however received little attention compared with maximal velocity kick biomechanics in rugby place kicking analyses.

Place kicking analyses in soccer have shown differences when kicking accurately and for maximum ball velocity. Godik et al. (1993) found that higher ball velocities were associated with the greatest level of accuracy in players who executed instep kicks at their own approach speed. Conversely, when soccer players were instructed to kick the ball with the highest possible velocity, higher approach velocities were linked to less accurate kicking mechanics. Lees and Nolan (1998) showed that when a player is instructed to perform a kick accurately there is a reduction in ball velocity and also in angular velocities of the lower extremity joints. Teixeira et al. (1999) found that soccer kicks aimed towards a defined target were associated with longer duration and smaller ankle displacement and velocity compared with kicks performed towards an undefined target. The research cited above suggests that the target determines the actual constraints on accuracy; its manipulation leads to a trade-off between speed and accuracy of the kick. In other words, when the player is required to perform an accurate kick, then the approach as well as the joint rotations and velocities are also lower compared with those recorded during a powerful kick.

Despite the depth of research in soccer-specific analyses, knowledge regarding rugby union place-kicking remains largely unexplored in biomechanics literature. Specifically, there does not appear to be any published information regarding the potential trade-off between accurate kicking and the generation of ball velocity. Therefore, this study aimed to identify biomechancal differences in rugby place kicking kinematics when kicking towards a specific target and for maximum velocity. A study of this nature may be of practical significance to place kickers and coaches, who wish to better understand the differences in mechanics under different constraints pertinent to successful kicking execution,

\section{Methods}


Ten male participants volunteered to take part in this investigation (age $22.4 \pm 1.2$ years; height $1.81 \pm 0.07 \mathrm{~m}$; body mass $86.1 \pm 4.2 \mathrm{~kg}$ ). All participants were regular place kickers at University first team level. All were free from musculoskeletal injury and provided written informed consent in accordance with the declaration of Helsinki. Ethical approval for this project was obtained from the School of Sport Tourism and Outdoors ethical committee at the University of Central Lancashire.

\section{Procedure}

Kinematic information was obtained using an optoelectric motion capture system with 8 cameras (Qualisys Medical AB, Gothenburg, Sweden) using a capture frequency of $500 \mathrm{~Hz}$. Each participant performed place kicks of a standard rugby ball (Gilbert Virtuo, size 5) into a net positioned $8 \mathrm{~m}$ away in two conditions. In the maximum velocity kicking condition participants were instructed simply to kick the ball as hard as they could into the net. In the accuracy constrained condition participants were instructed to kick the ball towards a $0.5 \mathrm{x}$ $0.5 \mathrm{~m}$ square positioned $5 \mathrm{~m}$ behind the net onto the wall of the biomechanics laboratory. The rugby ball was placed on a kicking tee positioned such that participants were able to adopt their preferred approach towards the ball. An additional four retroreflective markers were positioned onto the surface of the rugby ball, at one end of its longitudinal axis. Football contact was delineated using the initial displacement of these markers. Ten trials were obtained for each participant in each of the two conditions. The order in which participants performed in each of the conditions was counterbalanced.

The current investigation used the calibrated anatomical systems technique (CAST) to quantify angular kinematics (Cappozzo et al., 1995). To define the anatomical axes of the right and left feet, shanks and thigh segments, retroreflective markers were positioned bilaterally onto the calcaneus, 1st and 5th metatarsal heads, medial and lateral malleoli, medial and lateral epicondyle of the femur and greater trochanter. To delineate the pelvic segment co-ordinate axes, additional markers were positioned onto the anterior (ASIS) and posterior (PSIS) superior iliac spines. The hip joint centre was estimated using a regression technique based on the ASIS marker separation (Sinclair et al., 2013). Rigid carbon fiber 
tracking clusters with four non-linear retroreflective markers were positioned onto the pelvis, shank and thigh segments. Static calibration trials (not normalised to standing posture) were obtained for each participant to allow the anatomical positions of the retroreflective markers to be referenced in relation to the tracking clusters. Following the acquisition of the calibration trial markers not used for tracking were removed.

\section{Data-processing}

Discrete 3-D kinematic parameters were quantified using Visual 3-D (C-Motion Inc, Germantown MD, USA) and filtered at $15 \mathrm{~Hz}$ using a zero-lag low pass Butterworth 4th order filter. Joint rotations were created using an XYZ sequence of rotations referenced to coordinate axes created about the proximal end of the segment. 3-D kinematic measures from the hip, knee and ankle from the stance and kicking limbs which were extracted for statistical analysis were 1) angle at ball impact, 2) peak angle, 3) range of motion from stance limb contact to ball impact, 4) peak range of motion from foot contact to peak angle, 5) angular velocity at ball impact and 6) peak angular velocity. In addition to this linear ball velocity was also obtained. Finally, the duration of the kick phase from stance limb foot contact to ball impact and the medial-lateral distance from the kicking foot to the ball were extracted.

\section{Statistical analyses}

Means and standard deviations of 3-D kinematic parameters were calculated for each kicking condition. Differences in these parameters were examined using paired samples t-tests. The alpha criterion was adjusted using a Bonferroni adjusted alpha criterion $(\mathrm{p} \leq 0.0005)$ to control type I error. Effect sizes for all significant tests were quantified using partial eta squared $\left(\eta^{2}\right)$. Effect sizes were characterized in accordance with Cohen (1988), small $=0.2$, medium $=0.5$, and large $=0.8$. In accordance, with the Winter et al., (2014) an effect size of $>0.2$ was considered to be practically important. The data were also screened for normality using a Shapiro-Wilk which conformed that the normality assumption was met. Statistical analyses were conducted using SPSS 21.0 (SPSS Inc, Chicago, USA).

\section{Results}


Figures 1-4 present the mean 3-D angular kinematics of the hip, knee and ankle joints for the stance and kicking limbs. Tables 1-4 present the 3-D kinematic parameters from the hip, knee and ankle observed as a function of kicking condition.

\section{Velocity, distance and temporal measures}

The results showed that ball velocity was greater $\left(\mathrm{t}(9)=5.61, \mathrm{p}<0.0005, \eta^{2}=0.78\right)$ when kicking for maximum velocity $\left(28.6 \pm 2.3 \mathrm{~m} \cdot \mathrm{s}^{-1}\right)$ in comparison to accuracy $\left(25.0 \pm 2.6 \mathrm{~m} . \mathrm{s}^{-1}\right)$. Release angle was not different $\left(\mathrm{t}(9)=1.25, \mathrm{p}>0.0005, \eta^{2}=0.15\right)$ between maximum velocity $\left(27 \pm 7^{\circ}\right)$ and accurate $\left(29 \pm 4^{\circ}\right)$ kicks. Foot linear velocity was greater $(\mathrm{t}(9)=4.76$, $\left.\mathrm{p}<0.0005, \eta^{2}=0.78\right)$ when kicking for maximum velocity $\left(22.4 \pm 2.5 \mathrm{~m} . \mathrm{s}^{-1}\right)$ in comparison to accuracy $\left(19.2 \pm 3.0 \mathrm{~m} \cdot \mathrm{s}^{-1}\right)$. In addition, no $\left(\mathrm{t}(9)=3.91, \mathrm{p}>0.0005, \eta^{2}=0.63\right)$ differences were observed between maximum velocity $(0.38 \pm 0.04 \mathrm{~m})$ and accurate $(0.34 \pm 0.07 \mathrm{~m})$ for the horizontal distance from the plant foot to the ball. Finally, it was observed that there was no difference $\left(\mathrm{t}(9)=3.30, \mathrm{p}>0.0005, \eta^{2}=0.55\right)$ in the duration of the kick phase between maximum velocity $(0.13 \pm 0.02 \mathrm{~s})$ and accurate kicking $(0.11 \pm 0.02 \mathrm{~s})$.

\section{Kicking limb}

@@@igure 1 nearhere@@@

@@@Figure2nearhere@@@

@@@Table1nearhere@@@

@@@Table2nearhere@@@

The results show in the sagittal plane that peak hip flexion angular velocity was greater $(\mathrm{t}(9)$ $\left.=5.11, \mathrm{p}<0.0005, \eta^{2}=0.74\right)$ when kicking for maximal velocity when compared to accurate kicking. Furthermore, knee extension angular velocity at ball contact was also shown to be greater $\left(\mathrm{t}(9)=6.21, \mathrm{p}<0.0005, \eta^{2}=0.81\right)$ in the maximum velocity condition in comparison to the accuracy condition. Finally, it was revealed that that ankle was $(t)=5.78, p<0.0005$, $\left.\eta^{2}=0.79\right)$ more plantar-flexed in the maximum velocity condition compared to when kicking for accuracy. In the transverse plane the results indicate that in the accuracy condition that the 
197

ankle was $\left(\mathrm{t}(9)=5.09, \mathrm{p}<0.0005, \eta^{2}=0.74\right)$ more externally rotated at ball contact in comparison to kicking for maximum velocity. Finally, it was also shown that that the ankle transverse plane rotation angular velocity was different $\left(\mathrm{t}(9)=6.13, \mathrm{p}<0.0005, \eta^{2}=0.81\right)$ between the two conditions at ball contact, with the accuracy condition showing the ankle to be externally rotating and the maximum velocity showing the ankle to be internally rotating.

\section{Stance limb}

@@@Figure 3nearhere@@@ @@@Figure4nearhere@@@

@@@Table3nearhere@@@ @@@Table4nearhere@@@

The results show in the transverse plane that peak hip internal rotation was $(\mathrm{t}(9)=5.61$, $\left.\mathrm{p}<0.0005, \eta^{2}=0.78\right)$ greater when kicking for maximal velocity when compared to accurate kicking. Finally, it was also shown that peak ankle internal rotation was greater $(\mathrm{t}(9)=5.29$, $\mathrm{p}<0.0005, \eta^{2}=0.76$ ) when kicking for maximal velocity when compared to accurate kicking.

\section{Discussion}

The aim of the current investigation was to determine the 3-D kinematic differences in rugby place kicking kinematics when kicking towards a specific target and for maximum velocity. This represents the first investigation to compare the biomechanics of rugby place kicking when kicking for maximum velocity and when kicking for accuracy. 
With regards to ball velocity, the results of the current investigation are to be expected and show that kicking for maximum velocity is associated with greater ball velocity than when kicking for accuracy. This is supported by the increases in knee extension angular velocity and foot linear velocity at impact when kicking for maximum velocity. This observation supports the findings of Sinclair et al. (2014) who showed that knee extension velocity was correlated with ball velocity during rugby place kicking. The findings from the current investigation appear to support those from soccer in-step kicking analyses regarding the existence of a speed/accuracy trade-off (Plamondon \& Alimi, 1997; Teixeira et al., 1999) in that the alterations in kicking mechanics necessary to promote accuracy were used at the expense of maximising ball velocity.

In addition, it was also observed that peak hip flexion angular velocity was also greater when kicking for maximum velocity. This supports the notion that the velocity of the distal segments is resolved via a pattern of segmental interactions termed the proximal to distal sequence. Hip flexion angular velocity contributes to about $50 \%$ of the resultant angular velocity of the more distal segments (Putnam, 1993). During the second half of the kick movement the hip flexion velocity is reduced as the knee extension velocity increases. Although the thigh angular velocity is decreased in the latter part of the kick phase it is nonetheless important when ball velocity is desirable to generate greater hip flexion velocities in order to angular velocity to the distal segments.

A further significant observation occurred at the ankle in both sagittal and transverse planes. It was found that the ankle was more dorsiflexed and externally rotated at ball contact, when kicking for accuracy. This finding concurs with those from soccer based analyses which have documented similar findings in accurate kicks in comparison to when kicking for maximum velocity (Lees \& Nolan, 2002; Teixeira, 1999). It is hypothesized that this observation relates to the kicking mechanics used by participants when kicking for accuracy. In order to maximize accuracy participants may have used a more side foot technique by externally rotating the ankle in order to ensure ball contact with the medial aspect of the foot. This is supported by Levanon \& Dapena (1998) who theorized that side foot or kicking in soccer is employed when there is a requirement for precision. Increased dorsiflexion ensures that the contact point is closer to the metatarsals than the ankle. Kellis and Katis, (2007) showed that 
when the ball is kicked with the aspect of the foot near the ankle, the ball is released with a much greater linear velocity.

In modern rugby union a significant number of points are now secured from place kicking, making successful execution of place kicks vital to the final outcome. The current investigation has importantly characterized the mechanics of both accurate and high velocity rugby union place kicks. Therefore based on the findings from the current work, coaches and applied practitioners should be encouraged to emphasize the importance of generating high knee angular velocity in kickers who are striving to generate greater kicking distance. This may involve exercises which develop explosive power in the quadriceps muscle group which serve to extend the joint. Conversely in kickers who are seeking to improve the accuracy of their place kicks should be encouraged to focus on drills which promote increased dorsiflexion and external rotation of the foot at the instance of ball contact. This may ultimately enable kickers to combine these two key aspects and increase the likelihood of victory for their team.

A limitation of the current investigation is the laboratory based nature of the data collection procedures. Firstly, whilst accuracy was the focus of one of the experimental conditions it could not be specifically examined, rather the participants were instructed simply to aim for a specific target positioned onto the laboratory wall. In addition, the confines of the laboratory may also have affected the ecological validity of the ball release characteristics. Linthorne \& Stokes (2014) demonstrated in a field based experiment that the optimum projection angle is around $30^{\circ}$, with increasing projection angles leading to reductions in ball release velocity. Thus it appears that the trials examined during this study may not be truly not representative of a kick at goal. Future field based analyses may wish to consider the effects of accurate and maximum velocity kicking mechanics. A further limitation is the playing level of the participants used in this experiment. A sample of skilled yet not elite rugby union place kickers was tested as part of this investigation. This indicates that the observations may not be generalizable to populations outside this study. Future work should seek to quantify mechanical differences between accurate and maximum velocity kicks in more skilled place kickers. 
267 In conclusion, the current investigation has demonstrated that differences in kicking 268 mechanics exist when kicking for accuracy when compared to kicking for maximum velocity.

269 It is likely that kickers may have chosen utilize these mechanics when kicking for accuracy in 270 a deliberate attempt to improve precision, but at the expense of sacrificing ball velocity and 271 thus the distance that the ball can be kicked. Therefore, the specific findings from the current 272 work have implications for coaches and applied practitioners which may facilitate 273 improvements in kicking performance.

\section{References}

Baker, J., \& Ball, K. (1993). Biomechanical Considerations of the Drop Punt. Australian Conference of Science and Medicine in Sport, Melbourne, Sports Medicine Australia.

Baktash, S., Hy, A., Muir, S., Walton, T., \& Zhang, Y. (2009). The effects of different instep foot positions on ball velocity in place kicking, International Journal of Sports Science and Engineering, 3, 85-92.

Barfield, W.R. (1995). Effects of selected kinematic and kinetic variables on instep kicking with dominant and non-dominant limbs. Journal of Human Movement Studies, 29, 251-272.

Bezodis, N., Trewartha, G., Wilson, C. \& Irwin, G. (2007). Contributions of the non-kickingside arm to rugby place-kicking technique. Sports Biomechanics, 6, 171-186.

Cappozzo, A., Catani, F., Leardini, A., Benedeti, M.G., \& Della, C.U. (1995). Position and orientation in space of bones during movement: Anatomical frame definition and determination. Clinical Biomechanics, 10, 171-178.

Cohen, J. (1988). Statistical Power Analysis for the Behavioral Sciences. New York, NY: Routledge Academic.

Godik, M., Fales, I., \& Blashak, I. (1993). Changing the kicking accuracy of soccer players depending on the type, value and aims of training and competitive loads. In: Science and soccer II. Eds: Reilly, T., Clarys, J. and Stibbe, A. London: E\&FN Spon. 254-260.

Kellis, E., \& Katis, A. (2007). Biomechanical characteristics and determinants of instep soccer kick. Journal of Sports Science and Medicine, 6, 154-165. 
Lees, A., \& Nolan, L. (1998). The biomechanics of soccer: A review. Journal of Sports Sciences, 16, 211-234.

Levanon, J., \& Dapena, J. (1998). Comparison of the kinematics of the full-instep and pass kicks in soccer. Medicine and Science in Sports and Exercise, 30, 917-927.

Linthorne, N.P., \& Stokes, T.G. (2014). Optimum projection angle for attaining maximum distance in a rugby place kick. Journal of Sports Science and Medicine, 13, 211-216.

Plamondon, R., \& Alimi, A. M. (1997). Speed/accuracy trade-offs in target-directed movements. Behavioral and Brain Sciences, 20, 279-349.

Putnam, C. (1993). Sequential motions of body segments in striking and throwing skills: descriptions and explanations. Journal of Biomechanics, 26, 125-135.

Putnam, C.A., \& Dunn, E.G. (1987). Performance variations in rapid swinging motions: Effects on segment interaction and resultant joint moments. In: Jonsson, B. (Eds), Biomechanics X-B. Human Kinetics Publishers, Inc., Champaign, IL, 661 - 665.

Robertson, D.G.E., \& Mosher, R.E. (1985). Work and power of the leg muscles in soccer kicking. In Biomechanics IX-B. Eds: Winter, D.A., Norman, R.W., Wells, R.P., Hayes, K.C., and Patla, A.E. Champaign, IL: Human Kinetics Publishers, 533-538.

Sinclair, J., Edmundson, C.J, Brooks, D., \& Hobbs, S.J. (2011). Evaluation of kinematic methods of identifying gait Events during running. International Journal of Sports Science and Engineering, 5, 188-192.

Sinclair, J., Greenhalgh, A., Edmundson, C.J., Brooks, D. \& Hobbs, S.J. (2013). The influence of barefoot and barefoot-inspired footwear on the kinetics and kinematics of running in comparison to conventional running shoes. Footwear Science, 5, 45-53.

Sinclair, J., Taylor, P.J., Atkins, S., Bullen, J., Smith, A., \& Hobbs, S.J. (2014). The influence of lower extremity kinematics on ball release velocity during in-step place kicking in rugby union. International Journal of Performance Analysis in Sport, 14, 64-72.

Teixeira, L. (1999) Kinematics of kicking as a function of different sources of constraint on accuracy. Perceptual and Motor Skills, 88, 785-789. 
322 Winter, E.M., Abt, G., \& Nevill, A.M. (2014). Metrics of meaningfulness as opposed to 323 sleights of significance. Journal of Sports Sciences, 32, 901-902,

324 Zhang, Y., Guangyu, L., \& Shengquan, X. (2011). Movement sequences during instep rugby 325 kicking a 3D biomechanical analysis, International Journal of Sports Science and 326 Engineering, 6, 89-95.

\section{$328 \quad$ Figures}

329 Figure 1: Hip, knee and ankle joint kinematics from the kicking limb as a function of the dominant and non-dominant limbs (black $=$ max velocity $\&$ dash $=$ accuracy).

Figure 2: Hip, knee and ankle joint angular velocities parameters from the kicking limb as a function of the dominant and non-dominant limbs (black = max velocity $\&$ dash $=$ accuracy).

333 Figure 3: Hip, knee and ankle joint kinematics from the stance limb as a function of the 334 dominant and non-dominant limbs (black $=$ max velocity $\&$ dash $=$ accuracy).

335 Figure 4: Hip, knee and ankle joint angular velocities parameters from the stance limb as a 336 function of the dominant and non-dominant limbs (black $=$ max velocity $\&$ dash $=$ accuracy). 